\title{
PROPRIEDADES PSICOMÉTRICAS DO ISSL NO CONTEXTO DA SEGURANÇA PÚBLICA
}

\author{
Cristiane Faiad \\ Universidade de Brasília \\ Victor Souza \\ Universidade de Brasília
}

Lucas Heiki Matsunaga

Universidade de Brasília

Carlos Manoel Lopes Rodrigues

Universidade Católica de Brasília

Helena Rinaldi Rosa

Universidade de São Paulo

\begin{abstract}
Resumo
O objetivo deste estudo foi analisar as propriedades psicométricas do Inventário de Sintomas de Stress para Adultos de Lipp (ISSL) no contexto da Segurança Pública. Para tanto, o inventário foi aplicado em 786 profissionais da Segurança Pública de 18 estados brasileiros e de diversas instituições de segurança pública. Foram analisadas a discriminação dos itens do inventário pela teoria de resposta ao item e as dimensões fatoriais pela análise de correspondência múltipla. A partir da análise dos autovalores, do exame do diagrama de sedimentação e da conformidade com a teoria foi observada apenas uma dimensão para o instrumento, diferentemente da proposta de dois fatores até então indicada na literatura. Pela análise dos itens pela TRI, é sugerida a exclusão de três itens do instrumento, por não possuírem graus suficientes de discriminação. Ao final, os autores indicam uma agenda de pesquisa que busque identificar as propriedades psicométricas do ISSL em outros contextos.
\end{abstract}

Palavras-chave: estresse; segurança pública; evidências de validade.

\section{PSYCHOMETRIC PROPERTIES OF THE ISSL IN THE CONTEXT OF PUBLIC SECURITY}

\begin{abstract}
The aim of this study was to analyze the psychometric properties of the Lipp Adult Stress Symptom Inventory (ISSL) in the context of Public Security. For this purpose, the inventory was applied to 786 Public Security professionals from 18 Brazilian states and several public security institutions. We calculated the discrimination of inventory items by item response theory and factorial dimensions according to the multiple correspondence analysis. From the analysis of the eigenvalues, the scree plot and by the conformity with the theory, only one dimension was observed for the instrument, as opposed to two, as previously reported in the literature. By analyzing the items using IRT, we suggest the exclusion of three items from the instrument, as they do not have sufficient discrimination power. Finally, we suggest new study regarding the psychometric properties of ISSL in other contexts.
\end{abstract}

Keywords: stress, public security, validity evidence. 


\title{
PROPIEDADES PSICOMÉTRICAS DEL ISSL EN EL CONTEXTO DE LA SEGURIDAD PÚBLICA
}

\begin{abstract}
Resumen
El objetivo de este estudio fue analizar las propiedades psicométricas del Inventario de Síntomas de Stress para Adultos de Lipp (ISSL) en el contexto de la Seguridad Pública. Para ello se aplicó el inventario en 786 profesionales de la Seguridad Pública de 18 estados brasileños y de diversas instituciones de seguridad pública. Se analizaron la discriminación de los ítems del inventario por la teoría de respuesta al ítem y las dimensiones factoriales por el análisis de correspondencia múltiple. A partir del análisis de los autovalores, del examen del diagrama de sedimentación y de la conformidad con la teoría se observó sólo una dimensión para el instrumento, a diferencia de dos, como propuesto por la literatura. Por el análisis de los ítems por la TRI, se sugiere la exclusión de 3 ítems del instrumento, por no poseer grados suficientes de discriminación. Al final, los autores sugieren una agenda de investigación que busca identificar las propiedades psicométricas del ISSL en otros contextos.
\end{abstract}

Palabra clave: estrés, seguridad pública, validez

\section{INTRODUÇÃO}

O estresse é um conceito que teve origem na física, principalmente nos trabalhos de Robert Hooke, com estudos sobre tensão e deformações na matéria de estruturas por interferência de um causador externo. Contudo, expandiu-se por analogia para áreas da psicologia, psiquiatria e sociologia, abrangendo dimensões biológicas, sociais e psicológicas que afetam o ser humano em nível emocional (Lazarus, 1993).

Embora haja divergências no conceito do estresse entre diversas áreas da saúde, Lazarus (1993) propõe quatro dimensões essenciais para sua análise: (a) a relação entre pessoa e ambiente estressor, (b) a avaliação psicológica-cognitiva de um indivíduo a designar aquele agente como uma ameaça ou como algo benigno, (c) as estratégias de enfrentamento para lidar com a demanda estressora ou coping, (d) os padrões complexos de efeitos fisiológicos e cognitivos referidos como reações ao estresse. Assim, segundo o autor, seria necessário analisar os efeitos psicológicos da situação estressante, a antecipação que indivíduos possuem sobre uma situação estressora eminente, e os desafios que aquela demanda exige do indivíduo para que ele mobilize recursos e estratégias de enfrentamento.

Lazarus (2000) também afirma que o grau de reação ao estresse depende da avaliação cognitiva e as estratégias individuais de enfrentamento, além das influências ambientais e internas do organismo. Ou seja, se o indivíduo muda sua relação com o ambiente estressor ou sua percepção sobre a situação, as condições de estresse também podem mudar.

Assim, o estresse caracteriza-se por toda e qualquer reação do indivíduo que lhe cause alguma alteração de seu equilíbrio, na tentativa de se adaptar a um agente estressor. A consequência ou resposta a essa alteração varia de indivíduo para indivíduo, por envolver fatores não somente ambientais e físicos, mas também cognitivos (Joca, Padovan, \& Guimarães, 2003; Lipp \& Tanganelli, 2002; Margis, Picon, Cosner, \& Silveira, 2003), hormonais e psicológicos. O estresse será 
considerado prejudicial quando a exposição ao evento estressor é duradoura e se o indivíduo tem propensão ao desenvolvimento de alguma doença.

Especificamente no contexto de trabalho esta situação implica em agravos à saúde do trabalhador, maior risco de acidentes, queda da produtividade e aumento significativo dos custos da saúde pública (Hassard, Teoh, Visockaite, Dewe, \& Cox, 2017). Baptista e Carneiro (2011) encontraram em sua revisão de literatura que níveis elevados de estresse ou de ansiedade podem estar associados a sintomas físicos-dores de cabeça, diminuição de libido, insônia e fadiga, e mentais-redução da capacidade de concentração e preocupação excessiva, sintomas semelhantes àqueles desencadeados pela depressão. Observaram que fatores como mudanças de chefia, cargo, condição financeira, estratégias inadequadas de enfrentamento, dificuldades para lidar com o superior e horários de trabalho ininterruptos, por exemplo, são apontados como fatores de vulnerabilidade para o desenvolvimento de estresse laboral, ansiedade e depressão. Resultado similar foi encontrado por Kortum, Leka e Cox (2010) junto a especialistas em 21 países, com destaque para a relação entre sintomas físicos e mentais como consequência da exposição aos estressores no ambiente laboral.

No contexto da segurança pública, a avaliação deste construto alcança relevante análise, dada a natureza da tarefa policial. Profissão considerada altamente estressante, alia-se a uma vivência direta do profissional em um ambiente de crescente violência nas ruas incluindo confrontos sociais e riscos reais, que acarretam em consequências físicas e emocionais (Azevedo, 2017; Nascimento, Pimentel, \& Adaid-Castro, 2016). Estas consequências podem estar associadas à pressão social e à exigência de uma maior eficiência por parte destes profissionais (Collins, \& Gibbs, 2003; Costa, Accioly Junior, Oliveira, \& Maia, 2007; Faiad, Coelho Junior, Caetano, \& Albuquerque, 2012; Reis, \& Faiad, 2014; Santos, 2016; Thadeu, Ferreira, \& Faiad, 2012) e à falta de reconhecimento social e apoio do Estado (Souza, \& Minayo, 2017). Não obstante, a polícia se encontra como um dos grupos de ocupação de maior potencial para o desenvolvimento de estresse ocupacional e de doenças mentais de acordo com o Surveillance of Occupational Stress and Mental IIIness (Health and Safety Executive, 2016). Ainda outros autores a consideram a profissão mais estressante (Brito, \& Souza, 2004; Huddleston, Paton, \& Stephens, 2006).

A partir dessas diferentes temáticas, surge a necessidade de se planejar e elaborar pesquisas voltadas para uma leitura das especificidades, incluindo a construção de medidas que abarquem em sua amostra policiais e a verificação da validade dos instrumentos neste contexto (Reis \& Faiad, 2014). Conforme Azevedo (2017) aponta, há escassez de pesquisas científicas sobre esses profissionais, principalmente aquelas voltadas para a problematização de aspectos psicológicos que os permeiam, incluindo também estudos sobre medidas de estresse - fator emocional significativo ao trabalho policial. 
$\mathrm{Na}$ avaliação de sintomas de estresse, o Inventário de Sintomas de Stress para Adultos de Lipp (ISSL) tem sido amplamente utilizado para avaliação da existência de sintomas e na identificação do nível em que se encontram. Em estudos com profissionais de segurança pública, o ISSL tem sido o instrumento predominante na avaliação de estresse, por meio de dados apresentados de forma descritiva, em sua totalidade, considerando-se o resultado como um diagnóstico ou retrato do efetivo avaliado.

No primeiro estudo de validade do instrumento, realizado por Lipp e Guevara (1994), foi considerado para avaliação o que Lipp (2005) identificou como a validação empírica do instrumento. Neste estudo, os autores avaliaram dois diferentes grupos, sendo uma primeira amostra composta por 105 de pacientes, que procuraram um centro clínico de atendimento, e uma segunda composta por 124 estudantes universitários. Para a primeira amostra, procurou-se encontrar uma relação entre o diagnóstico dos pacientes realizado pela primeira autora e os resultados obtidos no inventário aplicado por outros pesquisadores nos mesmos indivíduos. Já para a última amostra os autores buscaram apresentar a relação entre a autoavaliação de estudantes sobre o estresse e os resultados obtidos ao preencherem o inventário. Os resultados do inventário mostraram que $84 \%$ das pessoas que procuraram o centro clínico e $68 \%$ dos universitários possuíam sintomas de estresse. Os autores afirmaram que houve uma perfeita correlação entre o diagnóstico realizado pela primeira autora e o resultado de estresse verificado pelo inventário.

Embora seja escassa a literatura sobre evidências de validade do ISSL, destacam-se estudos complementares entre Miguel e Noronha (2007) e Oswald (2009). No primeiro, encontrou-se poucas evidências de validade convergente entre o ISSL e a Escala de Vulnerabilidade ao Estresse no Trabalho, representada por uma correlação positiva baixa de $r=0,20, p<0,05$. O segundo autor, no entanto, encontrou uma correlação positiva moderada/alta, $r=0,42, p<0,01$. Além desses estudos, Cusatis Neto (2007) encontrou correlações positivas e significativas, porém baixas ou moderadas entre a maioria das variáveis da Escala de Estressores Ocupacionais e do ISSL

Em termos teóricos, Lipp (2005) definiu o estresse como a reação de um organismo, que contempla aspectos físicos, psicológicos, mentais e hormonais, desenvolvidos por meio de etapas ou frases. Segundo a definição deste trabalho, o estresse se desenvolve em quatro fases. Conforme Ganster (2008), as fases de estresse são identificadas a partir dos sintomas físicos e psicológicos identificados pelo respondente e pela duração destes sintomas, sendo um instrumento baseado em autorrelato. $O$ instrumento faz uso de duas estratégias de medida, com uma medida de estresse baseada no bem-estar psicológico e outra baseada em sintomas físicos. Ambas as medidas têm em comum certa facilidade para serem respondidas, mas também poucas referências às fontes de estresse, e requerem cuidado quando utilizadas em conjunto, pois corre-se o risco de se assumir que 
sintomas físicos e psicológicos possuem trajetórias etiológicas iguais (Ganster, 2008; Ganster, \& Rosen, 2013).

As fases de Alerta, Resistência e Exaustão já compunham o modelo trifásico de Selye (1951), proposto para explicar o desenvolvimento da Síndrome Geral de Adaptação. Contudo, Lipp (2005) incluiu a fase da Quase-exaustão como uma etapa de seu instrumento. Tanto o modelo trifásico de Selye (1951), quanto o quadrifásico de Lipp (2005) podem ser classificados como modelos baseados nas respostas fisiológicas e psicológicas provocadas pela exposição aos eventos estressores, com ênfase em um padrão de respostas que reflete a situação em que a pessoa está imersa (Glina, 2014).

As fases identificadas por Lipp (2005) são, então, Alerta, Resistência, Quase-exaustão e Exaustão. Para este modelo teórico, na primeira fase há intensa produção de adrenalina, que prepara o organismo para sobrevivência. Já na segunda fase, a Resistência, o organismo busca o equilíbrio interno para se adaptar. Nesta fase são descritas sensações de desgaste e cansaço. Na terceira fase, Quase-exaustão, há o adoecimento, deterioração e fragilização de órgãos. Por fim, a última fase, chamada de Exaustão, engloba doenças graves, que ocorrem nos órgãos mais vulneráveis.

Em um estudo no contexto da segurança pública, Costa et al. (2007) aplicaram o ISSL em 264 policiais militares da cidade de Natal, com o objetivo de diagnosticar a ocorrência e a fase de estresse e determinar a sintomatologia prevalente nos contextos físico e mental. Os autores descreveram uma proporção de $52,6 \%$ de policiais sem sintomas de estresse. Dos $47,4 \%$ com estresse, houve uma prevalência de $39,8 \%$ na fase de resistência, com sintomas psicológicos registrados em $76 \%$ dos policiais com estresse. Dentre as relações com outras variáveis, os autores indicaram correlação positiva entre o estresse e a variável sexo, indicando maior prevalência nas mulheres. Utilizado como diagnóstico, a pesquisa identifica a importância da construção de intervenções preventivas, por meio de ações de diagnóstico, orientação e controle do estresse.

Rossetti et al. (2008) realizaram uma pesquisa junto a 250 policiais federais, para avaliarem os níveis de estresse de policiais no ambiente de trabalho e a identificação de sintomatologias. Para isso fizeram uso do ISSL. Os autores mostram uma prevalência de policiais na fase da resistência e um maior nível de estresse em mulheres, sendo esta diferença relativa ao gênero frequente nas pesquisas sobre estresse (Lipp, 2009; Lipp, \& Tanganelli, 2002). Resultados semelhantes foram relatados por Lipp, Costa e Nunes (2017) em um estudo com 1.837 policiais militares, bombeiros, policiais civis, agentes da polícia técnica além de pessoal administrativo, em que a prevalência da fase de resistência foi constatada, com taxas significativas de sintomas de desgaste físico, alterações musculares, dificuldades de memória, insônia e cansaço mental.

A avaliação do estresse assume, assim, grande importância social e relevância em diferentes contextos de sua avaliação, requerendo estratégias que 
permitam alcançar a expressão do fenômeno nos diversos contextos em que se apresenta. Contudo, dentre os desafios encontrados em sua medida estão a multiplicidade de modelos teóricos e de técnicas de medida utilizadas (Ganster, 2008) e, no contexto da segurança pública, as peculiaridades dos estressores presentes no contexto do trabalho policial (Morash, Haarr, \& Kwak, 2006). E este último desafio retoma a importância em se discutir a evidência de validade da medida de estresse para esse contexto.

É importante discutir em que medida os instrumentos apresentam qualidades psicométricas e evidências de validade para seu uso adequado para qualquer contexto (Primi, Muniz \& Nunes, 2009). Hogan (2006), ao afirmar que os testes possuem especificidades contextuais para seu uso, ressalta que os mesmos não podem ser aplicados sem um objetivo específico e que corresponda com a validade e natureza daquele. Ou, mais precisamente, identificar qual a evidência que uma medida tem para determinado contexto, aproximando-se ao máximo do mundo real que se busca avaliar (Pasquali, 2017).

Além disso, essa avaliação psicométrica torna-se importante, uma vez que, segundo Noronha, Primi e Alchieri (2004), uma grande parcela dos instrumentos psicológicos comercializados no Brasil carece de fundamentação científica ou suas evidências de validade e precisão não são adequadamente relatadas e, portanto, necessitam de verificações de suas propriedades, por meio de técnicas adequadas de tratamento de dados. Já os autores Coelho Junior e Faiad (2012) ressaltam sobre a importância de se investigar a generalidade de uma medida, por meio de sua aplicação em amostras distintas de sua validação original, de forma a identificar a existência de invariância fatorial quando se diversifica o perfil amostral.

Neste sentido, o objetivo deste trabalho é analisar as qualidades psicométricas do ISSL no contexto de aplicação à Segurança Pública. Segundo Lipp (2005), pela análise de correspondências múltiplas, o ISSL enquadra-se em um modelo bifatorial, onde os itens são representados ou pelo fator físico ou pelo fator psicológico. E conforme a literatura, esse contexto de análise representa uma amostra que compartilha graus de estresse elevados.

\section{MÉTODO}

\section{Participantes}

A amostra foi composta por 786 profissionais da área de segurança pública, provenientes dos estados do Amapá, Bahia, Ceará, Distrito Federal, Espírito Santo, Goiás, Mato Grosso, Mato Grosso do Sul, Minas Gerais, Paraíba, Pernambuco, Piauí, Paraná, Rio de Janeiro, Rio Grande do Sul, Sergipe, São Paulo e Tocantins ( $n=18$ ). A idade média foi de 37,06 anos ( $D P=7,67$ ), variando de 21 a 67 anos, com prevalência de homens $(85,4 \%)$. Esta distribuição manteve a tendência da 
composição majoritariamente masculina do efetivo da área de segurança pública, como indicado pela Secretaria Nacional de Segurança Pública - SENASP (2017), que varia entre $73,3 \%$ e $92,3 \%$ de homens a depender da instituição. Quanto à escolaridade, a maioria dos participantes afirmou ter Ensino Superior Completo (39,6\%), com tempo de serviço médio de 12,10 anos e apenas $21,11 \%$ ocupando cargo de chefia. Dentre as instituições pesquisadas, encontra-se a Polícia Militar, a Guarda Civil Municipal, a Polícia Civil, a Polícia Científica e o Corpo de Bombeiros, sendo $12 \%$ da amostra proveniente de atividades administrativas, $45,2 \%$ de atividades operacionais, $41,1 \%$ tanto de atividade administrativa quanto operacional e $1 \%$ de atividade na área de saúde.

\section{Instrumento}

Foi utilizado o Inventário de Sintomas de Stress para Adultos de Lipp (ISSL; Lipp, 2005). Este instrumento foi construído com base no ISS, identificando o estresse pela fase da sintomatologia, seja ela somática ou psicológica. 0 instrumento possui um caderno de aplicação com 53 sintomas, sendo 37 itens somáticos e 19 psicológicos que diferem quanto a intensidade do estresse. 0 primeiro quadro se refere à fase alerta e compreende 12 sintomas físicos e 3 psicológicos experienciados nas últimas 24 horas. O segundo quadro subdivide-se em duas partes, a primeira abordando a fase de resistência e a segunda de quase exaustão com um total de 10 sintomas físicos e 5 psicológicos observados na última semana, sendo que não há uma distinção no escore entre o que se torna fase de resistência ou fase de quase-exaustão, que são apenas integrados como um único quadro. Já o terceiro quadro avalia a exaustão assinalando-se os principais sintomas apresentados no último mês, composta por 12 sintomas físicos e 11 psicológicos.

Escores brutos acima de 6 no quadro 1, 3 no quadro 2 e 8 no quadro 3 são indicativos diagnóstico positivo de estresse, segundo a autora. Caso os escores obtidos estejam acima dos limites nos três quadros é identificado processo de agravamento do estresse. Para definição da fase de estresse em que o sujeito se encontra, deve-se considerar o quadro em que o indivíduo mais pontuou, em termos percentuais.

Conforme estudos apresentados no manual do instrumento, o ISSL apresenta alfa de Cronbach de 0,91 para a escala geral, sendo que seus resultados permitem saber em qual fase do estresse a pessoa se encontra e se os sintomas que prevalecem são mais físicos ou psicológicos.

\section{Procedimentos}

Os dados foram coletados por meio de uma pesquisa de levantamento online. $O$ instrumento foi disponibilizado em software livre e posteriormente encaminhado, por e-mail, aos profissionais de segurança pública. Todos os participantes foram voluntários e assentiram sua participação e anuência com o 
Termo de Consentimento Livre e Esclarecido (TCLE). Esta pesquisa encontra-se inserida no projeto aprovado pelo comitê de ética em Pesquisa com Seres Humanos do Instituto de Psicologia da Universidade de São Paulo (parecer 1.736.565).

\section{Análise de Dados}

Para análise de dados, foram reproduzidas as análises de comparações múltiplas para dois fatores, e posteriormente um fator pelo IBM SPSS StatisticS 23. Ao final, foi realizada a análise de teoria de resposta ao item (TRI) com utilização do pacote Latent Trait Models under IRT (Itm, Rizopoulos, 2006), do software R.

\section{RESULTADOS}

Para análise de evidência de validade para o contexto em questão, foi realizada inicialmente uma análise descritiva do instrumento. A pontuação de cada quadro foi calculada conforme a instrução presente no manual (Lipp, 2005), sendo adicionado um ponto a cada marcação dos participantes. As médias e os desviospadrão de cada quadro e tipo de sintoma podem ser observadas na Tabela 1.

Tabela 1.

Estatísticas descritivas das respostas dos sujeitos aos diferentes quadros.

\begin{tabular}{ccccc}
\hline Quadro & Sintomas & Valor Máximo & Média & Desvio Padrão \\
\hline 1 & Físicos & 12,00 & 3,4648 & 2,51583 \\
& Psicológicos & 3,00 & 0,7335 & 0,77294 \\
\hline 2 & Físicos & 10,00 & 3,4246 & 2,24775 \\
& Psicológicos & 5,00 & 2,1449 & 1,46116 \\
\hline \multirow{2}{*}{3} & Físicos & 12,00 & 2,3966 & 1,98412 \\
& Psicológicos & 11,00 & 3,8691 & 2,95239 \\
\hline
\end{tabular}

Após esta análise, foi realizada uma análise fatorial exploratória, por meio da análise de correspondências múltiplas (ACM). A ACM analisa a estrutura fatorial para um grande número variáveis categóricas (Hair, Black, Babin, Anderson, \& Tatham, 2009). Além dos autovalores, este tipo de análise permite a estimação da inércia, uma medida da proporção de variância explicada pelo fator. O modelo compreendido por dois fatores revelou um fator com autovalor 11,067 e outro com 2,644 , explicando ao total 13,71 da variação. Assim, pelo critério de percentagem de variância (Hair \& et al., 2009), o modelo de dois fatores não garante significância prática, ou seja, não explicando uma porcentagem significativa da variância pelos limites satisfatórios previstos nas ciências sociais. O segundo fator obteve inércia 0,05 , enquanto a inércia média era 0,129 . Estes valores estão presentes na Tabela 2 . 
Tabela 2.

Dimensões extraídas a partir de análise de correspondência múltipla.

Dimensão

Variância contabilizada

\begin{tabular}{|c|c|c|c|c|}
\hline Dimensão & Alfa de Cronbach & Total (autovalor) & Inércia & \% de variância \\
\hline 1 & 0,927 & 11,067 & 0,209 & 20,881 \\
\hline 2 & 0,634 & 2,644 & 0,050 & 4,988 \\
\hline Total & - & 13,710 & 0,259 & - \\
\hline Média & 0,871 & 6,855 & 0,129 & 12,934 \\
\hline
\end{tabular}

Nota. A Média de Alfa de Cronbach tem como base o autovalor médio.

A solução encontrada que melhor se adequa à discriminação dos itens é a de um modelo de apenas um fator. A ACM calcula os autovalores posteriores apenas após a extração do primeiro fator, que obteve, em termos práticos, os mesmos parâmetros $(\lambda=11,067$, Inércia $=0,209)$.

Pelo critério do teste do diagrama de sedimentação também foi possível encontrar, de acordo com o gráfico das raízes latentes representado pela Figura 1, a extração de apenas um fator, pois logo depois desse fator, a variância começa a se tornar praticamente comum entre os fatores. Além disso, Cattell (1966) sugere que se busque o ponto em que haja uma larga distinção entre autovalores grandes e autovalores pequenos. Considerando o valor do primeiro autovalor de 11 , e do segundo de 2, com amplitude de 9 pontos, ou mais de quatro vezes o valor do segundo autovalor, define-se este momento entre um e dois, eliminando-se todos os fatores após o primeiro. 


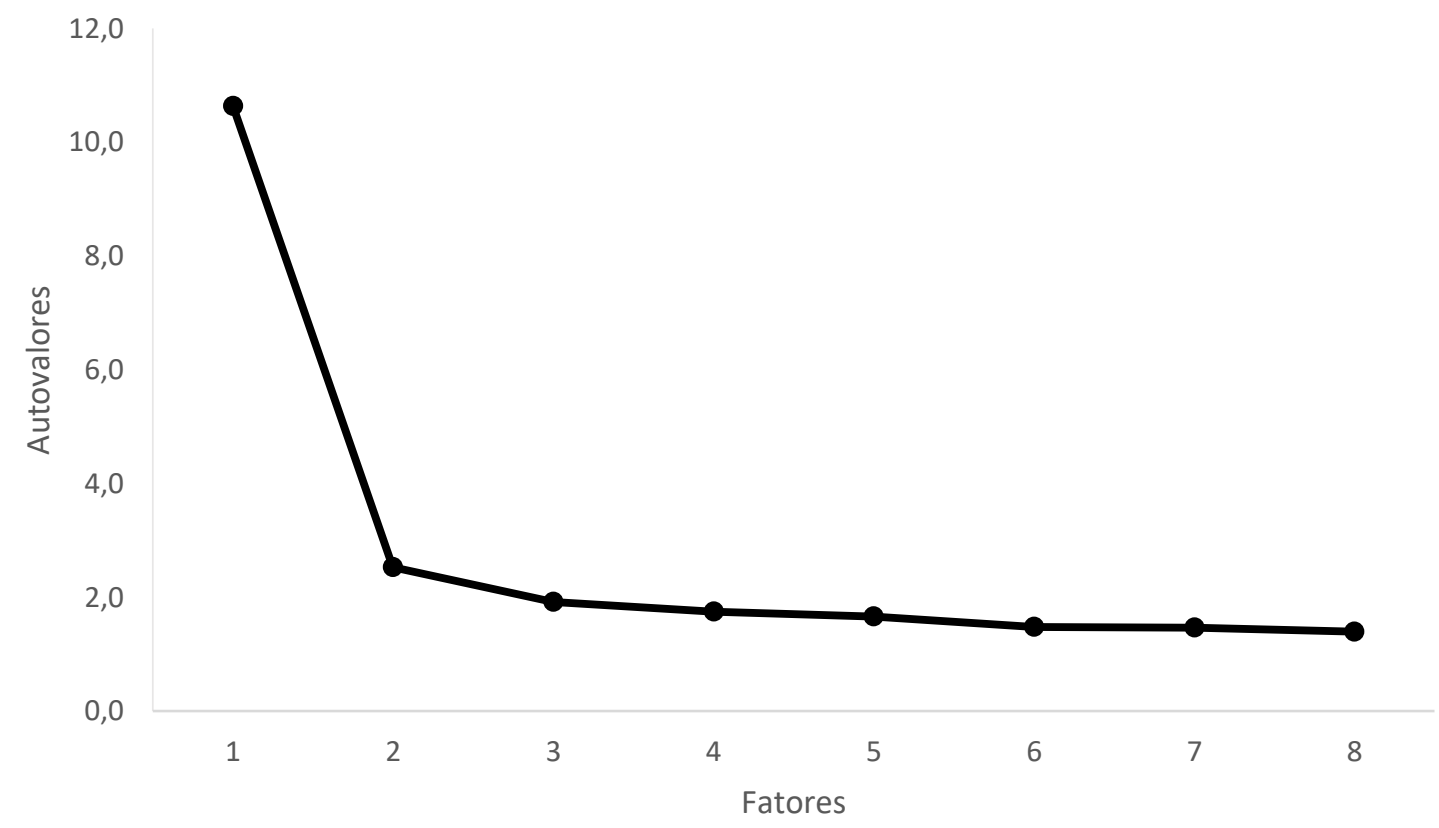

Figura 1.

Diagrama de sedimentação de 8 fatores na análise de correspondência múltipla.

Após a análise da estrutura fatorial, optou-se por se verificar a propriedade dos itens por meio da Teoria de Resposta ao Item (TRI), para análise da discriminação (a) e dificuldade (b) dos itens. A TRI foi escolhida para traçar o novo modelo, porque permite verificar a consistência individual dos itens de cada pessoa e determinar a variância do escore verdadeiro, do erro e do observador, ou seja, a TRI busca investigar uma consistência a partir de diversos critérios, estimando, pelo modelo de dois parâmetros, o grau de dificuldade e a discriminação dos itens (Hogan, 2006; Pasquali, \& Primi, 2003). Por meio desse modelo, foi possível averiguar que a função de informação do teste é distribuída normalmente em torno do valor 0 (log.Lik = -19162, AIC = 38536, BIC =39030), conforme a Figura 2 . Este formato da curva é desejável, visto que representa a maior quantidade de informação em torno do parâmetro médio da população, sendo capaz de melhor discriminar com precisão a maior parte dos indivíduos. Além disso, a curva se estende mais ao lado positivo do que ao negativo, indicando maior informação sobre os indivíduos de maior estresse - geralmente aqueles de interesse em situações clínicas. Os parâmetros dificuldade (b) e discriminação (a) do modelo de teoria de resposta ao item estão dispostos na Tabela 3, em conjunção com os parâmetros de discriminação da análise de correspondências múltiplas. 


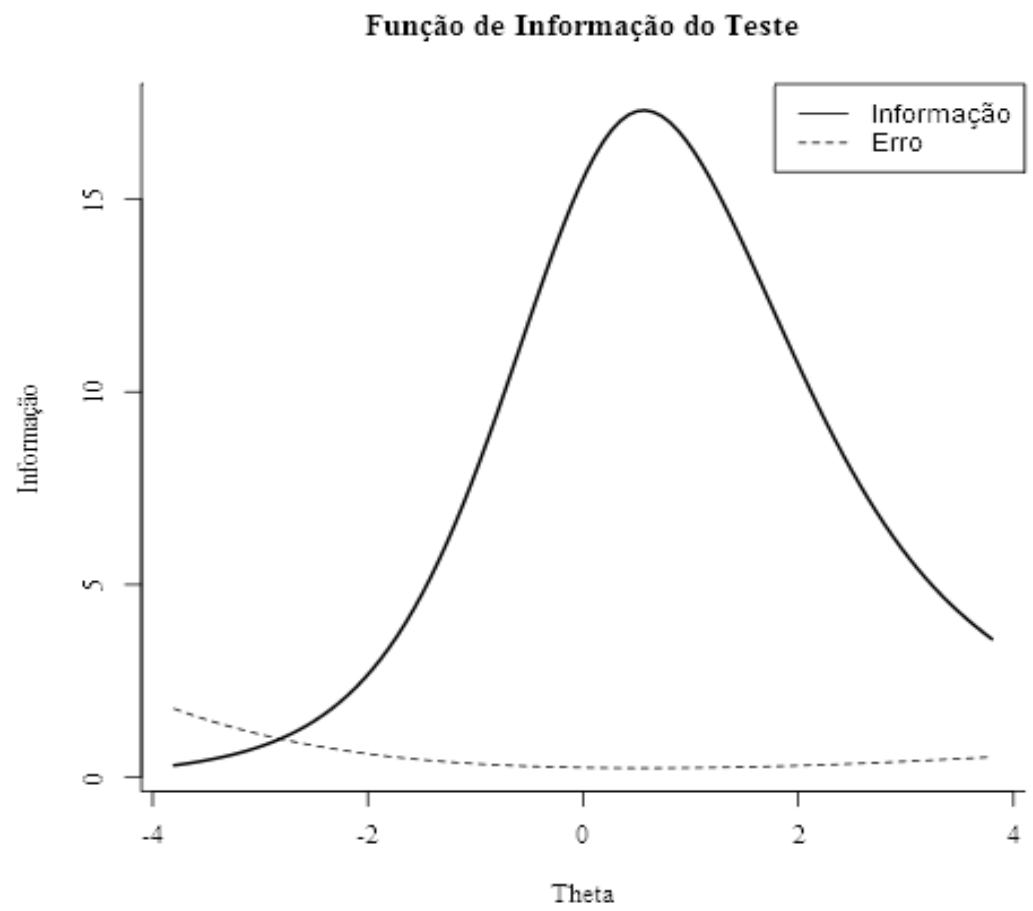

Figura 2.

Função de Informação do Inventário de Sintomas de Stress de Lipp com curva do Erro Padrão de Estimação (traçado).

Tabela 3.

Parâmetros dos itens do Inventário de Sintomas de Stress Lipp segundo encaixe por modelo de Teoria de Resposta ao Item e discriminação segundo encaixe da análise de correspondências múltiplas (ACM).

\begin{tabular}{cccccccc}
\hline Item & $\begin{array}{c}b \\
(\text { TRI })\end{array}$ & $\begin{array}{c}a \\
(\text { TRI })\end{array}$ & $\begin{array}{c}\text { Discriminação } \\
(\text { ACM })\end{array}$ & Item & $\begin{array}{c}b \\
(T R I)\end{array}$ & $\begin{array}{c}a \\
(T R I)\end{array}$ & $\begin{array}{c}\text { Discriminação } \\
(\text { ACM })\end{array}$ \\
\hline Q1F01 & 2,36 & 0,94 & 0,10 & Q2P13 & 0,58 & 0,86 & 0,148 \\
Q1F02 & 0,92 & 1,05 & 0,19 & Q2P14 & $-0,38$ & 1,72 & 0,309 \\
Q1F03 & 1,26 & 1,21 & 0,21 & Q2P15 & 0,82 & 1,29 & 0,251 \\
Q1F04 & 1,10 & 0,92 & 0,15 & Q3F01 & 2,59 & 0,97 & 0,091 \\
Q1F05 & $-0,39$ & 1,32 & 0,24 & Q3F02 & 1,29 & 1,33 & 0,224 \\
Q1F06 & 0,70 & 1,17 & 0,23 & Q3F03 & $-0,32$ & 1,39 & 0,254 \\
Q1F07 & 2,93 & 0,63 & 0,06 & Q3F04 & 1,82 & 1,42 & 0,183 \\
Q1F08 & $-0,28$ & 1,48 & 0,28 & Q3F05 & 1,54 & 1,52 & 0,223 \\
Q1F09 & 1,49 & 1,32 & 0,20 & Q3F06 & 2,80 & 0,78 & 0,075 \\
Q1F10 & 2,25 & 1,15 & 0,12 & Q3F07 & 1,43 & 1,09 & 0,176 \\
Q1F11 & 2,71 & 0,77 & 0,08 & Q3F08 & 1,17 & 1,42 & 0,245 \\
Q1F12 & 0,47 & 1,19 & 0,23 & Q3F09 & 0,67 & 0,77 & 0,123 \\
Q1P13 & 5,04 & 0,31 & 0,02 & Q3F10 & 1,48 & 1,55 & 0,228 \\
Q1P14 & 3,36 & 0,51 & 0,04 & Q3F11 & 2,82 & 0,99 & 0,086 \\
Q1P15 & 5,12 & 0,07 & $>0,01$ & Q3F12 & 3,31 & 3,63 & 0,023 \\
\hline
\end{tabular}




\begin{tabular}{cccccccc}
\hline Q2F01 & $-0,18$ & 1,31 & 0,25 & Q3P13 & 2,37 & 1,18 & 0,117 \\
Q2F02 & 0,72 & 1,84 & 0,34 & Q3P14 & 0,91 & 1,53 & 0,296 \\
Q2F03 & 1,64 & 1,13 & 0,17 & Q3P15 & 0,89 & 1,69 & 0,301 \\
Q2F04 & $-0,55$ & 1,69 & 0,29 & Q3P16 & 0,29 & 2,21 & 0,422 \\
Q2F05 & 0,63 & 1,39 & 0,28 & Q3P17 & 0,46 & 2,19 & 0,414 \\
Q2F06 & 1,15 & 1,04 & 0,18 & Q3P18 & $-0,43$ & 1,75 & 0,313 \\
Q2F07 & 2,99 & 0,66 & 0,06 & Q3P19 & 1,08 & 1,05 & 0,186 \\
Q2F08 & $-0,34$ & 1,56 & 0,29 & Q3P20 & $-0,01$ & 1,70 & 0,324 \\
Q2F09 & 2,52 & 1,15 & 0,11 & Q3P21 & 0,18 & 2,04 & 0,403 \\
Q2F10 & 0,97 & 1,49 & 0,28 & Q3P22 & 0,96 & 1,94 & 0,347 \\
Q2P11 & 0,01 & 1,82 & 0,35 & Q3P23 & 0,38 & 1,33 & 0,269 \\
Q2P12 & 0,77 & 1,40 & 0,27 & - & - & - & - \\
\hline
\end{tabular}

Os itens que não tiveram discriminação satisfatória, segundo a TRI (parâmetro b) foram Q1P13 ("aumento súbito de motivação"), Q1P14 ("entusiasmo súbito") e Q1P15 ("vontade súbita de iniciar novos projetos"), todos relacionados aos sintomas psicológicos. O item com maior grau de discriminação foi o Q3F12 ("enfarto"), de sintoma físico. Suas curvas características estão dispostas na Figura 3. 


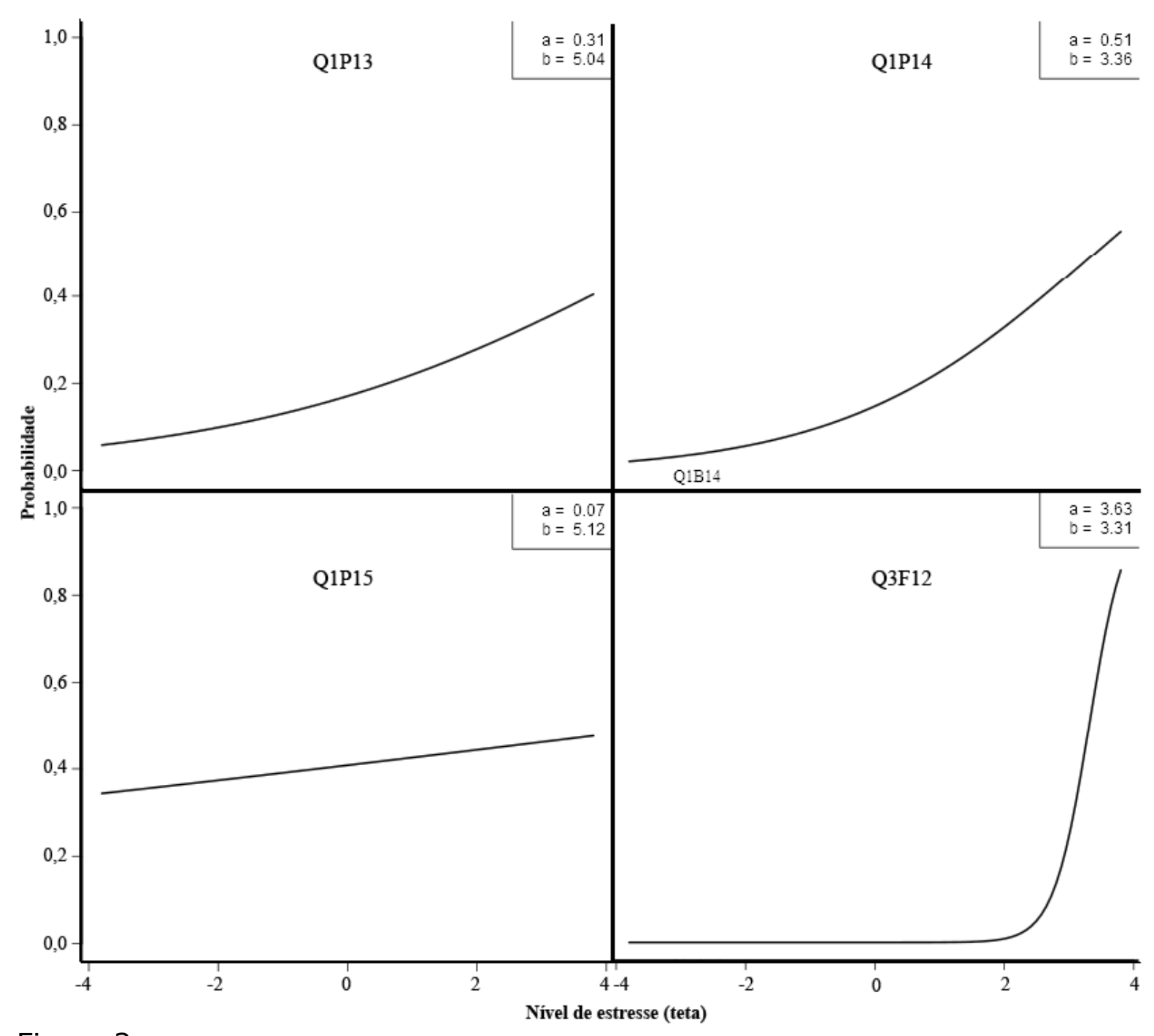

Figura 3.

Curvas características dos itens Q1P13, Q1P14, Q1P15 e Q3F12 com base no modelo de teoria de resposta ao item de dois parâmetros (2PL).

\section{DISCUSSÃO}

Blasius e Greenacre (2006) recomendam três critérios para análise da dimensionalidade dos dados na análise de correspondência múltipla. Os três critérios são (a) autovalores que expliquem mais do que a inércia média, (b) o exame do diagrama de sedimentação e (c) a conformidade com a teoria. Apesar desta recomendação ser feita para análise fatorial comum, Henson e Roberts (2006) sugerem a utilização de mais de um critério, em convergência, para realizar a escolha da dimensionalidade.

Assim, no presente trabalho, foi demonstrado que os três critérios propostos por Blasius e Greenacre (2006) convergem na decisão de retenção apenas um fator. Esta decisão é enfatizada adicionalmente visto que mesmo no modelo de dois fatores, o primeiro fator obteve coeficiente de fidedignidade considerado 
excelente (DeVellis, 2012), alpha de Cronbach $=0,927$, enquanto o possível segundo fator obteve alpha de apenas 0,634, considerado "questionável".

Considerando a conformidade da teoria, apesar de o Fator 2 separar apenas sintomas físicos dos demais, o Fator 1 agrupa uma grande quantidade de sintomas físicos com uma grande quantidade de sintomas psicológicos. Desta forma, não é possível sugerir que a divisão entre fatores físicos e psicológicos ocorra nesta possível dimensionalidade. Em outras palavras, a divisão em dois fatores não estaria em conformidade com a teoria.

Este resultado, aliado ao fato do instrumento se basear no rastreio de um padrão de respostas a exposição aos estressores, pode indicar que o padrão de respostas desta população específica possivelmente se difere do padrão de outras amostras. Conforme Glina (2014), isso pode ocorrer já que estressores específicos podem produzir padrões específicos de respostas. Nesta mesma linha, Ganster (2008) avalia que esse padrão nem sempre é facilmente identificável por medidas deste tipo.

Pode-se considerar como limitação deste estudo que não tenha sido realizada uma análise paralela. No entanto, para identificação da dimensionalidade de um instrumento, não há recomendações da realização de análise paralela quando se trata da análise de múltiplas correspondências. De qualquer forma, no presente trabalho, houve tentativa de realizar esta análise para avaliar a viabilidade da avaliação de dimensionalidade. Porém, em análise paralela, todos os fatores tiveram autovalor abaixo de um, tornando-a ininterpretável. Este fato pode ser o motivo pelo qual não há dados na literatura acerca do uso da análise paralela como método de definição de dimensionalidade no caso da análise de múltiplas correspondências.

A análise da discriminação pela Teoria de Resposta ao Item sugere a exclusão de três itens sobre sintomas psicológicos do inventário, por não possuírem propriedades psicométricas para discriminar indivíduos com estresse e sem estresse. Por se tratar de uma amostra relativamente alta de respondentes, essa decisão mostra-se adequada e pertinente à transformação de um novo modelo, uma vez que tais itens, por não apresentarem níveis satisfatórios de discriminação, não podem ser representativos dos objetivos propostos pelo instrumento. Os itens que não apresentaram discriminação adequada são Q1P13 ("aumento súbito de motivação"), Q1P14 ("entusiasmo súbito") e Q1P15 ("vontade súbita de iniciar novos projetos").

Os resultados sugerem uma agenda de pesquisa que busque identificar as propriedades psicométricas do Inventário de Sintomas de Stress de Lipp em outros contextos, bem como se verificar novos estudos que procurem por evidências de validade clínica (Tavares, 2003) do instrumento no contexto segurança pública. Ou seja, estudos que busquem a congruência entre informações independentes sobre um sujeito, que convergem sob uma mesma interpretação de seu estado clínico, conforme indicações nos estudos iniciais do ISSL. 
Considerando que sua estrutura fatorial diverge nesta amostra específica à apenas um fator se compararmos à proposta original do instrumento, há de se averiguar se realmente este inventário é um recurso discriminante de estresse em agentes da polícia na estrutura apresentada. Outra possível questão a ser levantada, seria a necessidade de maiores investimentos em adaptação ou construção de novas medidas de estresse para a segurança pública. Isto porque a avaliação de estresse neste contexto tem grande relevância, conforme discutido, devido às atividades e fatores de risco psicossociais enfrentados na atuação policial. Ademais, instrumentos dessa natureza são utilizados como subsídios para diagnósticos e intervenções institucionais, merecendo uma ampla discussão da evidência consequencial no uso desses dados.

Assim, estes resultados podem modificar o olhar de outros estudos que se utilizam deste instrumento neste contexto, além de novas sugestões teóricas de análise e avaliação dos escores obtidos em estudos anteriores. Isto porque as correlações entre todos os sintomas que deram origem a um único fator apontam para uma possível falha do instrumento em discriminar sintomas físicos e psicológicos, talvez por possuírem trajetórias etiológicas semelhantes (Ganster, 2008; Ganster \& Rosen, 2013).

\section{CONSIDERAÇÕES FINAIS}

A identificação dos estressores específicos a que estão expostos os profissionais de segurança pública, visto que estão submetidos a exigências profissionais únicas (Faiad et al., 2012), e em um contexto de desvalorização como o brasileiro (Souza \& Minayo, 2017) precisam ser aprofundadas. Para o presente instrumento, uma aplicação para análise de padrões de resposta específicos, de forma a mais acuradamente categorizar as diferentes formas como o estresse se manifesta nos indivíduos, são bem-vindos para aprimoramento do mesmo, bem como para o processo de revisão de uma teoria mais robusta sobre estresse.

Apesar de não ser provável, é certamente possível que a aplicação deste instrumento em outro contexto revele padrões diferentes de importância para os sintomas no contexto do estresse geral. Neste sentido, recomenda-se que análises sejam realizadas nestes outros contextos a fim de comparar os resultados e trazer mais robustez para a interpretação do instrumento aqui apresentada.

\section{DECLARAÇÃO DE CONFLITO DE INTERESSES}

Não há qualquer conflito de interesses. 


\section{REFERÊNCIAS}

Azevedo, A. C. F. (2017). Algemados ao stress: O eutress e o distress em agentes de segurança pública. (Dissertação de mestrado). Universidade do Porto, Portugal.

Baptista, M. N., \& Carneiro, A. M. (2011). Validade da escala de depressão: Relação com ansiedade e stress laboral. Estudos de Psicologia, 28(3), 345352. doi:10.1590/S0103-166X2011000300006

Blasius, J., \& Greenacre, M. (2006). Correspondence analysis and related methods in practice. In M. Greenacre \& J. Blasius (Eds.), Multiple correspondence analysis and related methods. Boca Raton, FL: Chapman \& Hall/CRC.

Brito, A. S. \& Souza, L. (2004). Representações sociais de policiais civis sobre profissionalização. Sociologias, 6(12), 304-327. doi:10.1590/S151745222004000200011

Cattell, R. B. (1966). The scree test for the number of factors. Multivariate Behavioral Research, 1, 245-276. doi:10.1207/s15327906mbr0102_10

Coelho Junior, F. A., \& Faiad, C. (2012). Evidências de validade da escala de satisfação no trabalho. Avaliação Psicológica, 11(1), 111-121.

Collins, P. A. \& Gibbs, A. C. C. (2003). Stress in police officers: a study of the origins, prevalence and severity of stress-related symptoms within a county police force. Journal of Occupational and Environmental Medicine, 53(4), 255263. doi:10.1093/occmed/kqg061

Costa, M., Accioly, Jr. H., Oliveira, J. \& Maia, E. (2007). Estresse: Diagnóstico dos policias militares em uma cidade brasileira. Revista Panamericana Salud Publica, 21(4), 217-222. doi:10.1590/S1020-49892007000300004

Cusatis Neto, R. (2007). Construção e validação da escala de estressores ocupacionais das linhas de produção. (Tese de doutorado). Pontifícia Universidade Católica, Campinas, Brasil.

DeVellis, R.F. (2012). Scale development: Theory and applications. Los Angeles, CA: Sage.

Faiad, C., Coelho Junior, F. A., Caetano, P. F., \& Albuquerque, A. S. (2012). Análise profissiográfica e mapeamento de competências nas instituições de segurança pública. Psicologia: Ciência e Profissão, 32(2), 388-403. doi:10.1590/S1414-98932012000200009

Ganster, D. C. (2008). Measurement challenges for studying work-related stressors and strains. Human Resource Management Review, 18(4), 259270. doi: $10.1016 /$ j.hrmr.2008.07.011

Ganster, D. C., \& Rosen, C. C. (2013). Work stress and employee health: A multidisciplinary review. Journal of Management, 39(5), 1085-1122. doi: $10.1177 / 0149206313475815$ 
Glina, D. M. R. (2014). Modelos teóricos de estresse e estresse no trabalho e repercussões na saúde do trabalhador. In D. M. R. Glina \& L. E. Rocha, (Eds.), Saúde Mental no Trabalho: Da teoria à prática (pp. 3 - 30). São Paulo: Roca.

Hair, J. F., Black, W. C., Babin, B. J., Anderson, R. E., \& Tatham, R. L. (2009). Análise multivariada de dados. Porto Alegre, RS: Bookman Editora.

Hassard, J., Teoh, K. R., Visockaite, G., Dewe, P., \& Cox, T. (2018). The cost of work-related stress to society: A systematic review. Journal of Occupational Health Psychology, 23(1), 1-17. doi:10.1037/ocp0000069

Health and Safety Executive (2016). Work related stress, anxiety and depression statistics in Great Britain 2016. Londres: HSE. Disponível em: http://www.hse.gov.uk/statistics/ acesso em 16 de agosto de 2017.

Henson, R. K., \& Roberts, J. K. (2006). Use of exploratory factor analysis in published research. Educational and Psychological Measurement, 66(3), 393416. doi:10.1177/0013164405282485

Hogan, T. P. (2006). Introdução à prática de testes psicológicos. São Paulo, SP: Grupo Gen-LTC.

Huddleston, L. M., Paton, D., \& Stephens, C. (2006). Conceptualizing traumatic stress in police officers: Preemployment, critical incident, and organizational influences. Traumatology, 12(3), 170-177. doi:10.1177/1534765606294911

Joca, S. R. L, Padovan, C. M., \& Guimarães, F. S. (2003). Estresse, depressão e hipocampo. Revista Brasileira de Psiquiatria, 25(supl. II), 46-51. doi:10.1590/S1516-44462003000600011

Kortum, E., Leka, S., \& Cox, T. (2010). Psychosocial risks and work-related stress in developing countries: Health impact, priorities, barriers and solutions. International Journal of Occupational Medicine and Environmental Health, 23(3), 225-238. doi:10.2478/v10001-010-0024-5

Lazarus, R. S. (1993). From psychological stress to the emotions: A history of changing outlooks. Annual Review of Psychology, 44(1), 1-22. doi:10.1146/annurev.ps.44.020193.000245

Lazarus, R. S. (2000). Toward better research on stress and coping. American Psychologist, 55(6), 665-673. doi:10.1037/0003-066X.55.6.665

Lipp, M. E. N. (2005). Manual do inventário de sintomas de stress para adultos de Lipp (ISSL). São Paulo, SP: Casa do Psicólogo.

Lipp, M. E. N. (2009). Stress and quality of life in Brazilian police officers: Differences in gender. Spanish Journal of Psychology, 12(2), 593-603. doi: $10.1017 /$ S1138741600001967

Lipp, M. E. N., Costa, K. R. D. S. N., \& Nunes, V. D. O. (2017). Estresse, qualidade de vida e estressores ocupacionais de policiais: Sintomas mais frequentes. Revista Psicologia Organizações e Trabalho, 17(1), 46-53. doi:10.17652/rpot/2017.1.12490

Lipp, M. E. N. \& Guevara, A. J. H. (1994). Validação empírica do Inventário de Sintomas de Stress. Estudos de Psicologia, 11(3), 43-49. 
Lipp, M. E. N., \& Tanganelli, M. S. (2002). Stress e qualidade de vida em magistrados da justiça do trabalho: Diferenças entre homens e mulheres. Psicologia: Reflexão e Crítica, 15(3), 537-548. doi:10.1590/S010279722002000300008

Margis, R., Picon, P., Cosner, Q. A. F., \& Silveira, R. O. (2003). Relação entre estressores, estresse e ansiedade. Revista de Psiquiatria, 25(1), 65-74. doi:10.1590/S0101-81082003000400008

Miguel, F. K., \& Noronha, A. P. P. (2007). Estudo dos parâmetros psicométricos da escala de vulnerabilidade ao estresse no trabalho. Revista Evaluar, 7, 1-18.

Morash, M., Haarr, R., \& Kwak, D. H. (2006). Multilevel influences on police stress. Journal of Contemporary Criminal Justice, 22(1), 26-43. doi:10.1177/1043986205285055

Nascimento, T. G., Pimentel, C. E. \& Adaid-Castro, B. G. (2016). Escala de atitudes frente à arma de fogo (EAFAF): Evidências de sua adequação psicométrica. Psicologia: Teoria e Pesquisa, 32(1), 239-248. doi:10.1590/01023772201602187239248

Noronha, A. P. P., Primi, R., \& Alchieri, J. C. (2004). Parâmetros psicométricos: Uma análise de testes psicológicos comercializados no Brasil. Psicologia: Ciência e Profissão, 24(4), 88-99.

Oswaldo, Y. C. (2009). Vulnerabilidade ao estresse no trabalho, coping, depressão e qualidade de vida: Evidências de validade. (Tese de doutorado). Universidade São Francisco, Itatiba, Brasil.

Pasquali, L. (2017). Validade dos testes. Examen: Pesquisa em Avaliação, Certificação e Seleção, 1(1), 14-48.

Pasquali, L. \& Primi, R. (2003). Fundamentos da teoria da resposta ao item. Avaliação Psicológica. 2(2), 99-110.

Primi, R., Muniz, M., \& Nunes, C. H. S. S. (2009). Definições contemporâneas de validade de testes psicológicos. In C. S. Hutz (Ed.), Avanços e polêmicas em avaliação psicológica (pp. 243-265). São Paulo, SP: Casa do psicólogo.

Reis, M. S. \& Faiad, C. (2014). Escala de Instabilidade Emocional Para a Segurança Pública. Psico-USF, 19(1), 87- 96. doi:10.1590/S1413-82712014000100009

Rizopoulos, D. (2006). Itm: An R package for latent variable modeling and item response theory analyses. Journal of Statistical Software, 17(5), 1-25. doi:10.18637/jss.v017.i05

Rossetti, M. O., Ehlers, D. M., Guntert, I. B., Leme, I. F. A. S., Rabelo, I. S. \& Delpgs, S. M.V. (2008). O Inventário de Sintomas de Stress para adultos de Lipp (ISSL) em servidores da Polícia Federal de São Paulo. Revista Brasileira de Terapias Cognitivas, 4(2), 108-119. doi:10.5935/1808-5687.20080018

Santos, A. P. (2016). The effects of legal judgments on psychological evaluations in the Brazilian Federal Police. Policing, 10(2), 113-120. doi: 10.1093/police/pav053 
Secretaria Nacional de Segurança Pública. (2017). Perfil das instituições estaduais de segurança pública 2013 (ano-base 2013). Brasília, DF: Ministério da Justiça.

Selye, H. (1951). The general-adaptation-syndrome. Annual Review of Medicine, 2(1), 327-342. doi:10.1146/annurev.me.02.020151.001551

Souza, E. R. D., \& Minayo, M. C. D. S. (2017). Public security in a violent country. Cadernos de Saúde Pública, 33(3). doi:10.1590/0102-311×00036217

Tavares, M. (2003). Validade clínica. PsicoUSF, 8(2), 125-136.

Thadeu, S. H., Ferreira, M. C., \& Faiad, C. (2012). A avaliação psicológica em processos seletivos no contexto da segurança pública. Avaliação Psicológica $11(2), 229-238$.

Sobre os autores

Cristiane Faiad é Professora Adjunta do Departamento de Psicologia Social e do Trabalho e do Programa de Pós-Graduação em Psicologia Social, do Trabalho e das Organizações, da Universidade de Brasília (UnB). Mestre e Doutora pela UnB. crisfaiad@gmail.com.

Victor Souza é doutorando no Programa de Pós-Graduação em Psicologia Social, do Trabalho e das Organizações da Universidade de Brasília.

victor.souza@msn.com.

Lucas Heiki Matsunaga é Graduando em Psicologia pela Universidade de Brasília. lucas.matsunaga@gmail.com.

Carlos Manoel Lopes Rodrigues é Mestre e Doutorando em Psicologia Social, do Trabalho, e das Organizações pela Universidade de Brasília.

prof.carlos.manoel@gmail.com.

Helena Rinaldi Rosa é Professora Doutora no Departamento de Psicologia da Aprendizagem, do Desenvolvimento e da Personalidade do Instituto de Psicologia da Universidade de São Paulo. hrinaldi@usp.br.

A contribuição de cada autor pode ser atribuída como se segue: C.F., V. S. e L. H. contribuíram para conceitualização, investigação, análise de dados e visualização do artigo; C.F., V. S., L. H. e C.M. realizaram a redação inicial do artigo(rascunho) e C.M. e H. R. foram responsáveis pela redação final (revisão e edição).

Recebido em: 08/09/2017

$1^{\text {a }}$ revisão em: $22 / 01 / 2018$

Aceito em: $16 / 06 / 2018$ 